\title{
Top quark pair production in association with a $Z$-boson at NLO accuracy
}

\author{
A. Kardos and Z. Trócsányi \\ Institute of Physics, University of Debrecen, \\ H-4010 Debrecen P.O.Box 105, Hungary and \\ Institute of Nuclear Research of the Hungarian Academy of Sciences, Hungary ${ }^{\text {}}$ \\ C.G. Papadopoulos \\ NCSR Demokritos, Institute of Nuclear Physics, Athens, Greece
}

(Dated: November 2, 2018)

\begin{abstract}
We present predictions for the production cross section of t-quark pair production in association with a $Z$ boson at the next-to-leading order (NLO) accuracy using matrix elements obtained from the HELAC-Oneloop package. We use the subtraction method for computing the radiative corrections as implemented in the POWHEG-Box, which was also used in several other computations of similar complexity.
\end{abstract}


In the recent years we have been witnessing the $N L O$ revolution in computing QCD jet cross sections. Before the millenium, one-loop amplitudes were computed analytically, which was a serious bottleneck for computing QCD radiative corrections to processes of final states with high multiplicity. Indeed, the most complex computations involved threejet production with hadrons in the initial state [1, 2] and electron-positron annihilation into four-jets [3]. The emergence of unitarity-based, fully numerical approaches to computing one-loop amplitudes [4-11] has changed the scope of possible computations completely. By now there are publicly available computer programs for this purpose [12, 13], which paved the way to the automation of NLO computations [14].

In this letter we present predictions for the process $p p \rightarrow \mathrm{t} \overline{\mathrm{t}} Z$ at the NLO accuracy in QCD. This process is interesting from the point of view of measuring the $t \bar{t} Z$ couplings at the LHC [15], which has not yet been measured directly. This coupling is predicted by the Standard Model (SM). Significant deviation from this predicted value could be possible signal of new physics beyond the SM. In order to optimize such measurements precise theoretical predictions are needed both for the signal and the background processes.

Our present computations constitute steps in an ongoing project for generating event samples for $p p \rightarrow \mathrm{t} \overline{\mathrm{t}}+X$ processes, where $X$ is a hard partonic object in the final state [16, 17. These event files are stored according to the Les Houches accord [18], and can be interfaced to standard shower Monte Carlo programs to produce predictions for distributions at the hadron level that are exact up to NLO accuracy upon expansion in the strong coupling. With such predictions at hand one can optimize the selection cuts for the signal process for improved experimental accuracy of the coupling measurements.

In our project we use the POWHEG-Box [19], which produces a general framework also to NLO computations based on the FKS subtraction scheme [20]. The box requires the relevant matrix elements as external input. For this purpose we use the HELAC-NLO package [21, 22], from which we obtain the matrix elements in a semi-automatic way. Although these tools have already been used successfully for many computations, the complexity of the problem requires tedious checking and validation procedures to make sure that the event samples can be safely used in experimental analyses. Part of this procedure is a careful check of the predictions at the NLO accuracy which is the subject of this letter.

We performed our calculations using the POWHEG-Box which requires the following ingredients: 
- Flavor structures of the Born $(g g \rightarrow Z t \bar{t}, q \bar{q} \rightarrow Z t \bar{t}, \bar{q} q \rightarrow Z t \bar{t})$ and real radiation $(q \bar{q} \rightarrow Z t \bar{t} g, g g \rightarrow Z t \bar{t} g, \bar{q} g \rightarrow Z t \bar{t} \bar{q}, g \bar{q} \rightarrow Z t \bar{t} \bar{q}, \bar{q} q \rightarrow Z t \bar{t} g, q g \rightarrow Z t \bar{t} q, g q \rightarrow Z t \bar{t} q)$ subprocesses $(q \in\{u, d, c, s, b\})$.

- The Born-level phase space was obtained by using the invariant mass of the t $\overline{\mathrm{t}}$-pair and four angles.

- Crossing invariant squared matrix elements with all incoming momenta for the Born and the real-emission processes were built using amplitudes obtained from HELAC-Oneloop [23] and HELAC-PHEGAS [24], respectively. The matrix elements in the physical channels were obtained by crossing.

- The color matrices for the color-correlated squared matrix elements were taken from HELAC-Dipoles [25].

- We used the polarization vectors to project the helicity amplitudes to Lorentz basis for writing the spin-correlated squared matrix elements.

With this input POWHEG-Box can be used to perform the necessary integrations numerically. In order to ensure the correctness of the computations, we performed comparisons of the matrix elements to those obtained from independent sources and consistency checks.

We compared the tree-level (Born and real-emission) matrix elements to those obtained from MADEVENT [26], while the matix element for the virtual correction, including the first three terms in the Laurent expansion in the dimensional regularisation parameter $\epsilon$ was compared to that from GoSam [13] in randomly chosen phase space points. In all cases we found agreement up to at least 6 digits. We also computed various distributions at leading order with both PowHel (=POWHEG-Box+HELAC-NLO) and MADEVENT and found agreement.

The consistency between real-emission, Born, color-correlated and spin-correlated matrix elements was checked by taking the soft- and collinear limits of the real-emission squared matrix elements in all possible kinematically degenerate channels using randomly chosen phase space regions. As a further consistency check, we have also implemented the similar process $p p \rightarrow \mathrm{t} \overline{\mathrm{t}} \gamma$, which has also been computed at the NLO accuracy [28, 29], and we found agreement with the predictions of Ref. [29]. The important difference from the point of view of the computations lies in the mass of the vector boson. In this respect the $\mathrm{t} \overline{\mathrm{t}} \gamma$-production resembles $t \overline{\mathrm{t}}+$ jet -production presented in Ref. [16], while $\mathrm{t} \overline{\mathrm{t}} Z$-production resembles $\mathrm{t} \overline{\mathrm{t}} H$ production, presented in Ref. [17]. 


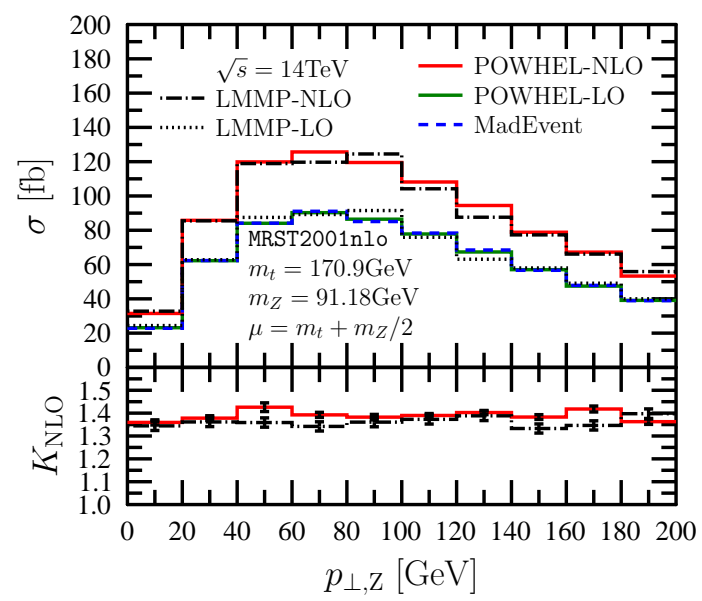

FIG. 1. Transverse momentum distribution of the $Z$-boson from Ref. [30] (LMMP) and from our calculation (PowHel). The lower panel shows our prediction for the NLO $K$-factor compared to that of LMMP.

For $t \bar{t} Z$ hadroproduction the fully inclusive cross section and the distribution of the transverse momentum of the $Z$-boson is the only published prediction at NLO accuracy. In Ref. [30] the prediction was made with the default scale choice $\mu\left(=\mu_{R}=\mu_{F}\right)=\mu_{0}(=$ $\left.m_{t}+m_{Z} / 2\right)$. With this scale and using the same input parameters we find agreement for the LO prediction $(0.808 \mathrm{pb})$, including the dependence on the scale $\mu$. However, at NLO accuracy our prediction is slightly larger, $(1.121 \pm 0.002)$ pb compared to $1.09 \mathrm{pb}$ [31] in Ref. [30], leading to an inclusive $K_{\text {inc }}=1.39$ (instead of the $K_{\text {inc }}=1.35$ in Ref. [30]).

For the $p_{\perp, Z}$-distribution we checked our LO prediction with MADEVENT, and found agreement as shown in Fig.1, but not with the LO predictions of Ref. [30]. As a result, the predictions of the two computations disagree also at NLO. In order to separate the effect of the differences at LO, in the lower panel we plot the $K$-factor of our computation against the $K_{\mathrm{NLO}}$ of Ref. [30]. The two predictions agree within the uncertainties of the numerical integrations except in two bins.

The almost constant value of the $K$-factor for the $p_{\perp}$-distribution of the $Z$-boson made the authors of Ref. [30] expect that the shape of many other kinematic distributions will also be approximately unchanged by NLO corrections. Our findings do not support this expectation. As an example, we show the $p_{\perp}$-distribution of the t-quark in Fig. 2 .

Next we turn our attention to make predictions for $\operatorname{t} \bar{t} Z$ hadroproduction at the current LHC. The parameters of our calculation were the following: $\sqrt{s}=7 \mathrm{TeV}$, CTEQ6.6M PDF 


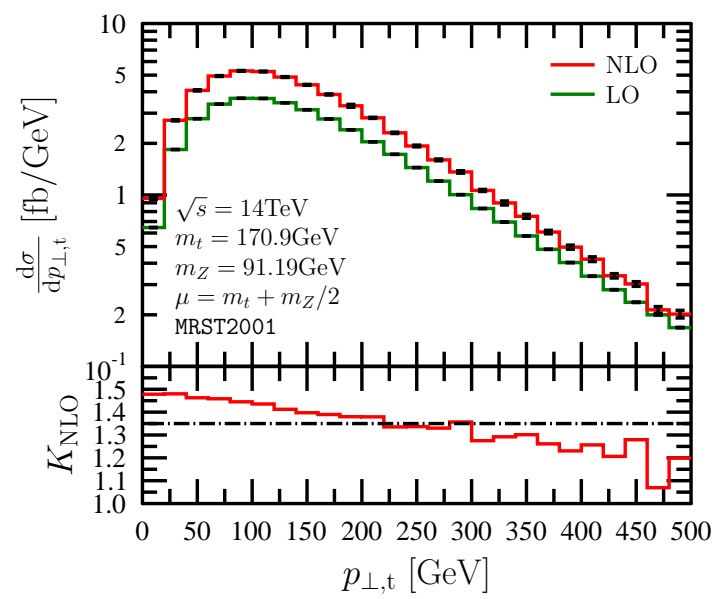

FIG. 2. Transverse momentum distribution of the t-quark. The lower panel shows our prediction for the NLO $K$-factor compared to a constant value $K_{\text {inc }}=1.39$.

set [32] from LHAPDF, 2-loop running $\alpha_{s}$, with $\Lambda_{5}^{\overline{\mathrm{MS}}}=226 \mathrm{MeV}, m_{t}=172.9 \mathrm{GeV}, m_{Z}=$ $91.1876 \mathrm{GeV}, m_{W}=80.399 \mathrm{GeV}, G_{F}=1.16639 \cdot 10^{-5} \mathrm{GeV}^{-2}$, the renormalization and factorization scales were chosen equal to $\mu_{0}=m_{t}+m_{Z} / 2$.

In Fig. 3 we show the transverse momentum distributions of the $Z$-boson and the tquark. We find that the $K$-factor for the $p_{\perp, Z}$-distribution is somewhat smaller and less uniform than that at $14 \mathrm{TeV}$. The bands correspond to the simultaneous variation of the renormalization and factorization scales between one half and two times the default value. We observe a significant reduction of the scale dependence as going from LO to NLO, also seen for other distributions. The extra radiation, present in the NLO computation, makes the spectra softer as expected. This softening decreases the $K$-factor below one for very large values of transverse momentum of the t-quark. Around the value of $p_{\perp}$ where the width of the NLO band shrinks to zero accidentally, and the band is unlikely to represent the effect of the missing higher order contributions.

In Fig. 4 we show the rapidity distributions of the $Z$-boson and the t-quark. The corrections are clearly not uniform, but increase towards large rapidities, making the predictions less reliable in those regions. For central rapidities the corrections are moderate and the scale uncertainty decreases significantly.

Finally, Fig.5 shows distributions of the separation in the rapidity-azimuthal angle plane

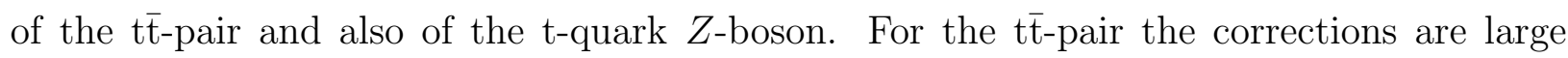
except for the back-to-back configuration, while for the $t Z$-separation the corrections are 

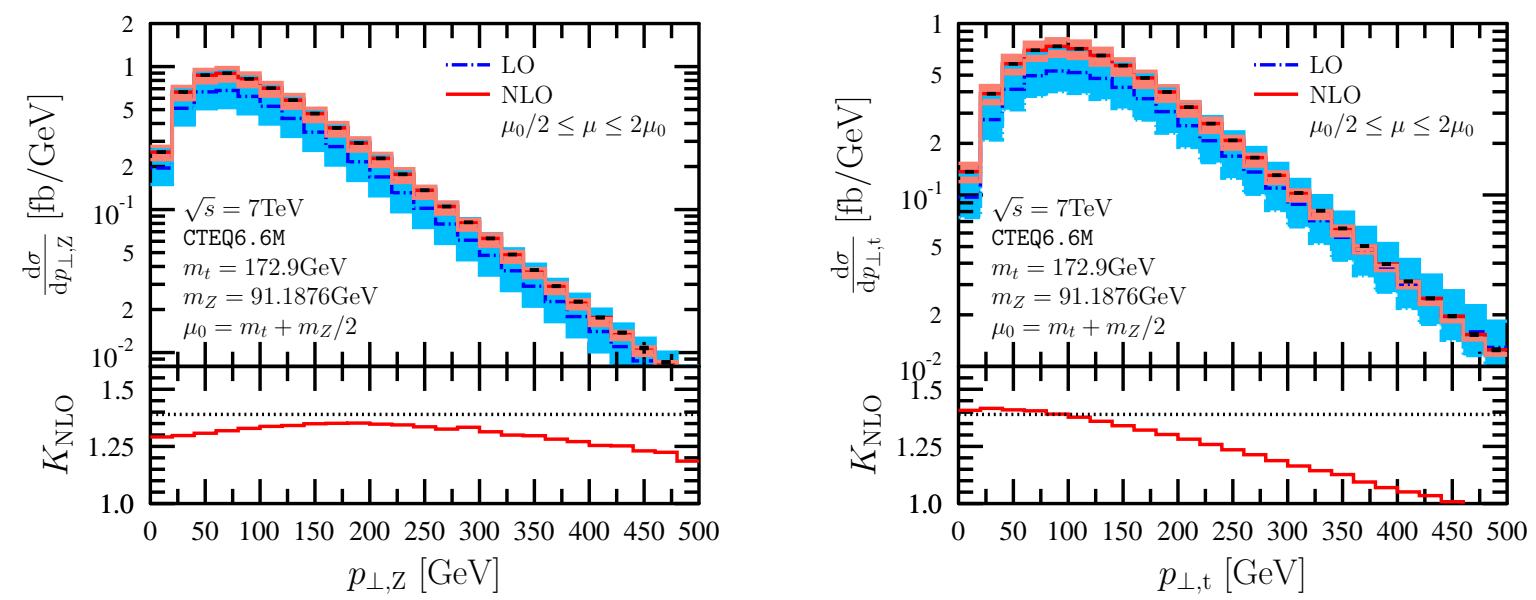

FIG. 3. Transverse momentum distributions of the $Z$-boson (left) and the t-quark (right). The lower panels show our prediction for the NLO $K$-factors compared to the constant value $K_{\text {inc }}=1.39$ found at $14 \mathrm{TeV}$ and default scale $\mu_{0}$.
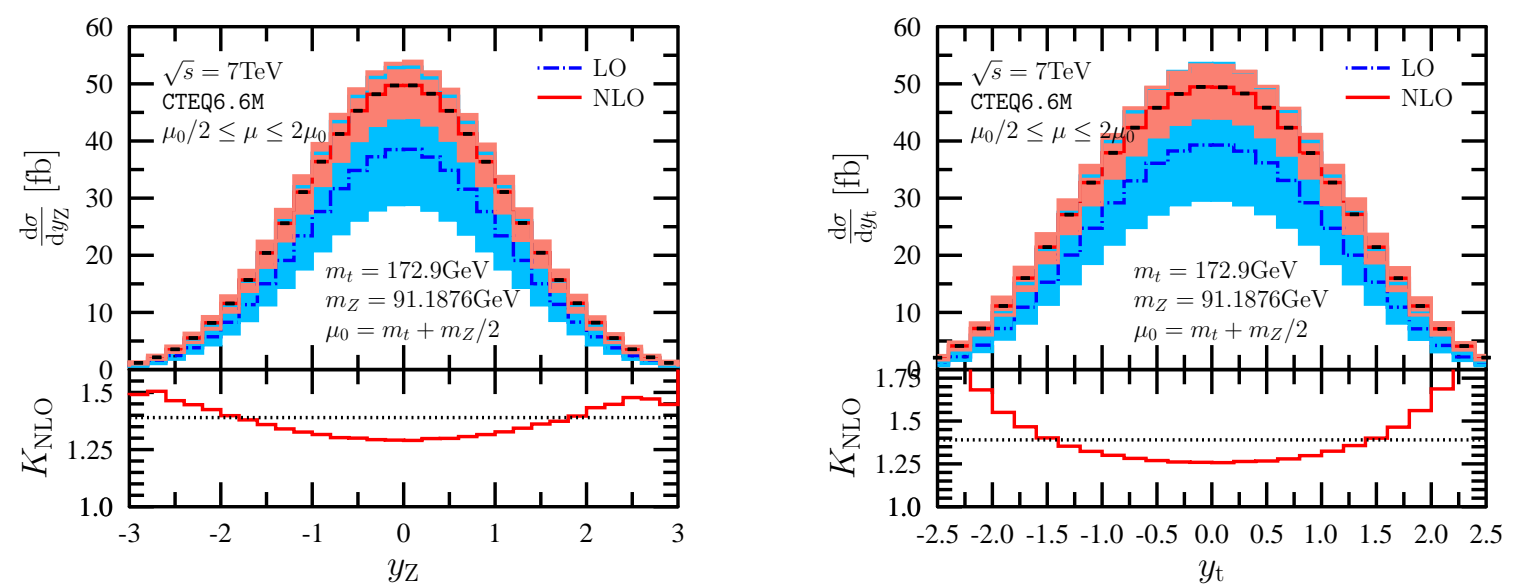

FIG. 4. Rapidity distributions of the $Z$-boson (left) and the t-quark (right). The lower panels show our prediction for the NLO $K$-factors compared to the constant value $K_{\text {inc }}=1.39$ found at $14 \mathrm{TeV}$ and default scale $\mu_{0}$.

moderate except for large rapidities.

In this letter we studied the hadroproduction of a $t \bar{t}$-pair in association with a $Z$ boson. This process is of interest for measuring the $t \bar{t} Z$-coupling directly at the LHC. Our predictions slightly disagree with the results of the only published computation. The difference most likely originates from the lower numerical precision of the integrations in Ref. [30].

We produced predictions for the LHC. In general one can conclude that the NLO predictions are sizeable, but not alarmingly large, lie in the 30-40\% range except towards the 

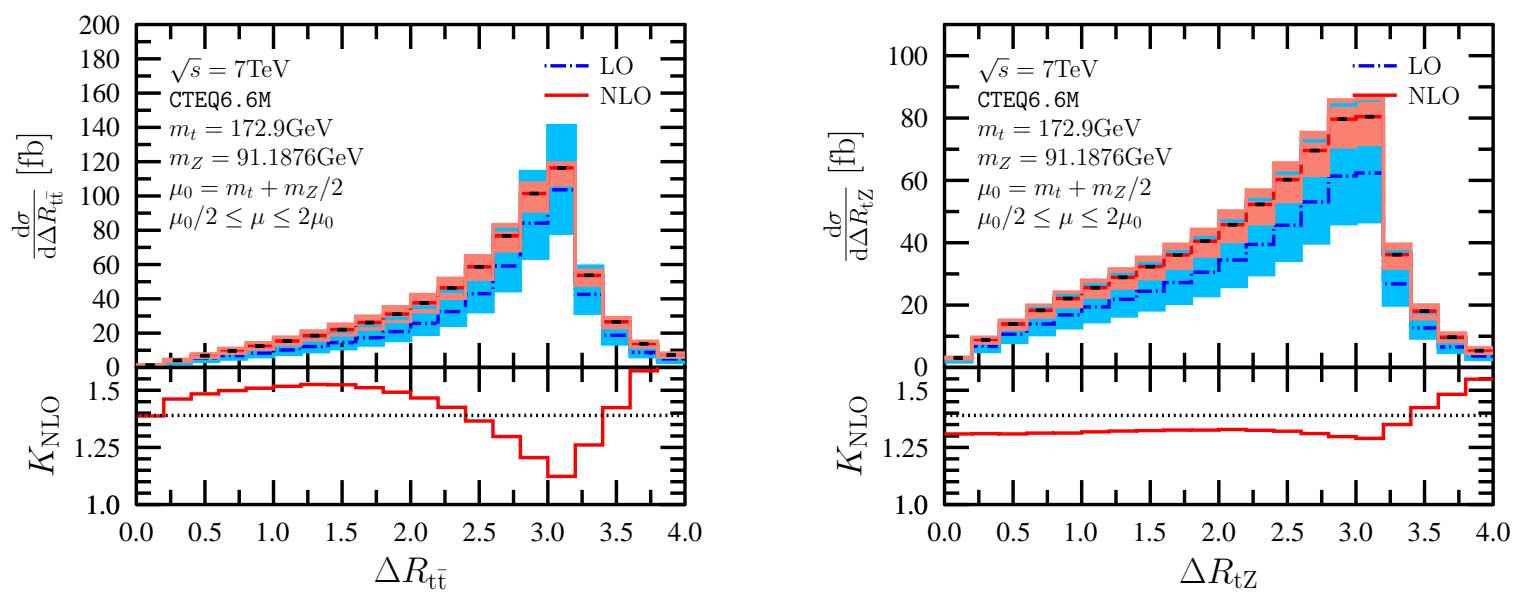

FIG. 5. Distributions of the $R$-separation between the $\mathrm{t} \overline{\mathrm{t}}$-pair (left) and between the t-quark and $Z$-boson (right). The lower panels show our prediction for the NLO $K$-factors compared to the constant value $K_{\text {inc }}=1.39$ found at $14 \mathrm{TeV}$ and default scale $\mu_{0}$.

edges of the kinematically available regions, where the $K$-factor can grow above 1.5 . As a result the dependence on the renormalization and factorization scales decreases significantly and the theoretical prediction becomes fairly reliable.

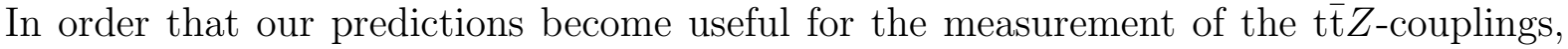
matching with shower Monte Carlo programs is desirable, which we consider in a separate publication. With such a matching we can let the t-quarks decay and study the optimization of the experimental cuts realistically.

This research was supported by the HEPTOOLS EU program MRTN-CT-2006-035505, the LHCPhenoNet network PITN-GA-2010-264564, the Swiss National Science Foundation Joint Research Project SCOPES IZ73Z0_1/28079, and the TÁMOP 4.2.1./B-09/1/KONV2010-0007 project. Z.T. thanks the Galileo Galilei Institute for Theoretical Physics for the hospitality and the INFN partial support during the completion of this work. We are grateful to F. Tramontano for comparing our one-loop amplitudes to results of GoSam, to F. Petriello for clarifying the numerical uncertainties in Ref. [30], and to the NIIFI Supercomputing Center for access to their computers.

* A. Kardos: adamkardos@inp.demokritos.gr

[1] Z. Nagy and Z. Trócsányi, Phys. Rev. Lett. 87, 082001 (2001) arXiv:hep-ph/0104315. 
[2] Z. Nagy, Phys. Rev. D 68, 094002 (2003) arXiv:hep-ph/0307268].

[3] Z. Nagy and Z. Trócsányi, Phys. Rev. D 59, 014020 (1999) [Erratum-ibid. D 62, 099902 (2000)] arXiv:hep-ph/9806317.

[4] Z. Bern, L. J. Dixon, D. C. Dunbar and D. A. Kosower, Nucl. Phys. B 435, 59 (1995) arXiv:hep-ph/9409265.

[5] A. Brandhuber, S. McNamara, B. J. Spence and G. Travaglini, JHEP 0510, 011 (2005) arXiv:hep-th/0506068].

[6] C. Anastasiou, R. Britto, B. Feng, Z. Kunszt and P. Mastrolia, JHEP 0703, 111 (2007) arXiv:hep-ph/0612277.

[7] G. Ossola, C. G. Papadopoulos and R. Pittau, Nucl. Phys. B 763, 147 (2007) arXiv:hepph/0609007].

[8] R. K. Ellis, W. T. Giele and Z. Kunszt, JHEP 0803, 003 (2008) arXiv:0708.2398 [hep-ph]].

[9] Z. Bern, L. J. Dixon and D. A. Kosower, Annals Phys. 322, 1587 (2007) arXiv:0704.2798 [hep-ph]].

[10] G. Ossola, C. G. Papadopoulos and R. Pittau, JHEP 0805, 004 (2008) arXiv:0802.1876 [hep-ph]].

[11] P. Draggiotis, M. V. Garzelli, C. G. Papadopoulos and R. Pittau, JHEP 0904, 072 (2009) arXiv:0903.0356 [hep-ph]].

[12] G. Ossola, C. G. Papadopoulos and R. Pittau, JHEP 0803, 042 (2008) arXiv:0711.3596 [hep-ph]].

[13] P. Mastrolia, G. Ossola, T. Reiter and F. Tramontano, JHEP 1008, 080 (2010) arXiv:1006.0710 [hep-ph]].

[14] V. Hirschi, R. Frederix, S. Frixione, M. V. Garzelli, F. Maltoni and R. Pittau, JHEP 1105, 044 (2011) arXiv:1103.0621 [hep-ph]].

[15] U. Baur, A. Juste, D. Rainwater and L. H. Orr, Phys. Rev. D 73, 034016 (2006) arXiv:hep$\mathrm{ph} / 0512262$.

[16] A. Kardos, C.G. Papadopoulos and Z. Trócsányi, Phys. Lett. B 750, 76 (2011) arXiv:1101.2672 [hep-ph]].

[17] M. V. Garzelli, A. Kardos, C. G. Papadopoulos and Z. Trócsányi, Europhys. Lett. 96, 11001 (2011) arXiv:1108.0387 [hep-ph]].

[18] J. Alwall et al., Comput. Phys. Commun. 176, 300 (2007) arXiv:hep-ph/0609017. 
[19] S. Alioli, P. Nason, C. Oleari and E. Re, JHEP 1006, 043 (2010) arXiv:1002.2581 [hep-ph]].

[20] S. Frixione, Z. Kunszt and A. Signer, Nucl. Phys. B 467, 399 (1996) arXiv:hep-ph/9512328.

[21] G. Bevilacqua et al., Nucl. Phys. Proc. Suppl. 205-206, 211 (2010) arXiv:1007.4918 [hep-ph]].

[22] G. Bevilacqua et al., arXiv:1110.1499 [hep-ph].

[23] A. van Hameren, C. G. Papadopoulos and R. Pittau, JHEP 0909, 106 (2009) arXiv:0903.4665 [hep-ph]].

[24] A. Cafarella, C. G. Papadopoulos and M. Worek, Comput. Phys. Commun. 180, 1941 (2009) arXiv:0710.2427 [hep-ph]].

[25] M. Czakon, C. G. Papadopoulos and M. Worek, JHEP 0908, 085 (2009) arXiv:0905.0883 [hep-ph]].

[26] F. Maltoni and T. Stelzer, JHEP 0302, 027 (2003) arXiv:hep-ph/0208156.

[27] G. Heinrich (on behalf of the GoSam collaboration), presentation at the ACAT 2011 conference, London.

[28] P. F. Duan, W. G. Ma, R. Y. Zhang, L. Han, L. Guo and S. M. Wang, Phys. Rev. D 80, $014022(2009)$.

[29] K. Melnikov, M. Schulze and A. Scharf, Phys. Rev. D 83, 074013 (2011) arXiv:1102.1967 [hep-ph]].

[30] A. Lazopoulos, T. McElmurry, K. Melnikov and F. Petriello, Phys. Lett. B 666, 62 (2008) arXiv:0804.2220 [hep-ph]].

[31] The estimated uncertainty is about $1.5 \%$ (F. Petriello, private communication).

[32] P. M. Nadolsky, H. -L. Lai, Q. -H. Cao, J. Huston, J. Pumplin, D. Stump, W. -K. Tung, C. -P. Yuan, Phys. Rev. D78, 013004 (2008). arXiv:0802.0007 [hep-ph]]. 